\title{
Who's There? Supporting Audience Awareness in Creative Content Systems
}

\author{
Eric Cook ${ }^{1}$, Stephanie Teasley ${ }^{1}$, Peggy Wong ${ }^{1}$, Max Kolasinski ${ }^{2}$, Bailey M. Waterman ${ }^{1}$ \\ ${ }^{1}$ School of Information; ' 2 Department of Computer Science and Engineering \\ University of Michigan \\ Ann Arbor, Ml 48109-2185 \\ [ericcook | steasley | pypeggy | mkolas | baileymw]@umich.edu
}

\begin{abstract}
In this poster abstract, we motivate and describe the design rationale for an approach to making audiences of creative content more visible and salient to producers contributing to user generated content and social media systems. Set in the specific context of digital photography, we discuss how this approach is appropriate for visualizing multiple audience types, thus supporting heterogeneous use models, relationship types and motivations. We also discuss the research questions and future assessment work through which this UI intervention will be evaluated.
\end{abstract}

\section{Keywords}

Photography, design, HCI, evaluation, creativity, audience, user-generated content, social media

\section{INTRODUCTION}

Consider examples of creative performance and presentation that occur in face-to-face settings: concerts, recitals, talks, gallery displays. In these and similar settings, the creator/performer's awareness of the audience is direct and visceral, deriving from proximity in a specific location. Through this co-location, a creator can derive a wealth of deeply contextualized information about his/her audience, either in reference to particular individuals ("look, Professor Smith is sitting the back row; I can't believe he is here.") or in reference to social group characteristics ("looks like the usual crowd turned up.") Explicit feedback or commentary is not required on the part of audience member to convey this information, just visibility.

Looking across a broader scope of human communicative activity, whether designated as explicitly "creative" or not, we know from literature in social and linguistic psychology

This is the space reserved for copyright notices.

ASIST 2011, October 9-13, 2011, New Orleans, LA, USA. Copyright notice continues right here. that individuals will adapt their communicative behavior in response to their perceived models of what intended recipients know and believe (Fussell \& Krauss, 1989; Nickerson, 1999; Newman-Norlund, et al, 2009). We build common ground (Clark \& Brennan, 1991; Clark, 1996) with whom we interact with, coordinating on process and content to build mutual interpretability. We leverage shared history and frames of reference to add contextual value and personal meaning into specific utterances or content (Cook \& Teasley, 2011; Cook, 2011). In literature on HCI, one location where these themes become visible is in the concept of designing for social translucence (Erickson \& Kellogg, 2000); we take this concept as a central point of inspiration in this work.

Such processes of message design and common ground formation require an awareness of the audience, of "who is in the room," metaphorically speaking. Yet audience information in user-generated media systems (now frequently utilized for the widespread spread of a wide variety of creative content) tends to be available only through aggregate log-data statistics or visible by feedback requiring explicit activity (comments, ratings, and so forth). These are useful metrics, but we assert, do not present a full picture of a creator's audience.

We take this as space as an opportunity for design and research, particularly in reference to utilizing IT to facilitate creative activity, a growing concern for technology researchers over the past decade (Shneiderman, Fischer, et al, 2005; Mitchell et al, 2003). Thus in this project, we investigate two main research questions:

RQ1: How does a more a more granular awareness of audience members - both those who have expressly left feedback and those that have not - affect creators contributing to user-generated media systems?

RQ2: When is this type of intervention most helpful; for which creator motivations, audience relationships or audience management practices?

\section{RESEARCH CONTEXT: DIGITAL PHOTOGRAPHY}

We chose to situate our investigation in a particular domain of creative activity, that of digital photography. Beyond the pragmatic need to scope and focus the project, photography 
presents a variety of interesting characteristics. It is a setting in which technology plays an influential role on production and dissemination, but is also informed by more than a century of norms, standards and conventions. Similarly, it is a context in which a wide range of skills, motivations and audience relationships are at play. As such, this resonated with the project goal to attend to a wide set of creators, professionals and serious amateurs as well as those engaged in more everyday and quotidian acts of creativity.

\section{DESIGN RATIONALE}

Our design rationale was driven by two high-level goals. First, we wanted to address the multiple types of audiences identified in our preliminary interviews, without overly privileging any one type. Second, we wanted to contextualize our design within an existing system's UI, in order to focus user reactions more specifically on our design intervention, and to ground our design within a realistic set of constraints.

\section{Multiple Audiences, Multiple Needs}

In prior work (Cook \& Teasley, 2011; Cook, 2011), we detailed the findings from interviews conducted during the first phase of this project, during which we described the perceived target audiences and audience management practices of photographers engaged in online photo sharing. In particular, recurrent audience types included: the self as audience, intimates (friends and family), photo subjects and event participants, communities of interest, communities of practice, professional contacts and peers, current and potential commercial clients, the generalized audience of "the internet." While we do not claim that this list is exhaustive of all possible audiences envisioned by digital photographers, it serves as directive to the scope and diversity of audience models and relationships that needed to be supported.

We also documented cases in which multiple audiences overlapped, and others where the photographer intentionally addressed multiple audiences simultaneously with the same image. As such, we wanted to ensure that we did not assume in our design that we knew a priori for any given user what their audience(s) would be for any given photo. Rather, our goal was to build a lightweight design that would support each user's process of deriving necessary contextual cues about their audience, such that it would be most useful for them at that moment in time.

\section{Contextualizing the Design Intervention}

As noted, we chose to evaluate our proposed design intervention within the interface of an existing site. In this case, we chose the online photo-sharing system Flickr.com. This decision was made for three primary reasons. First, while social networking systems such as Facebook do include photo-sharing as part of their suite of functions, Flickr is much more singularly structured around photography. This allowed us to focus our research participants' attention specifically within the domain of interest to the study. Second, Flickr continues to maintain an active and diverse user base. This facilitates the recruitment of research participants who are familiar with the existing functions and interface of the system, focusing their reactions more specifically on our proposed intervention. Third, Flickr provides API access to much of the public data on the system. Such data access makes the proposed intervention more realistic to develop as a thirdparty application, such as a Firefox plugin, while also presenting us with a more realistic set of constraints to drive our design. As will be discussed later, Flickr API access also allows our research team to create user-specific mockup interfaces for evaluation, presenting users with details from their own photostreams and contact lists in order to contextualize the evaluation process.

\section{Design Intervention}

Our proposed design was developed during the first half of 2011, via an iterative design process. We contracted a graphic designer to ensure that mockups matched the existing design conventions within Flickr. As we progressed through iterations on the design, we compared proposed features both with data from the preliminary interview accounts as well with the constraints of the Flickr API and system. In the end, we settled on the design presented in this abstract and poster.

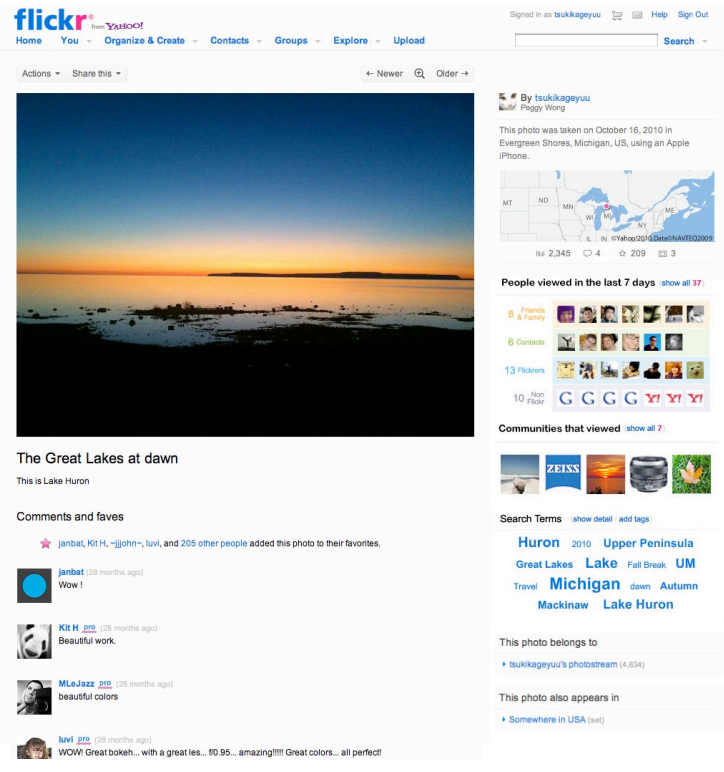

\section{Figure 1: Overview image, showing audience information (right column) in context of existing Flickr UI}

The primary conceit of the proposed design is the ability to track individual movement through the system, in order to facilitate the display of audience "footprints" left behind. While this is not currently implemented at this stage of the project, it is a feasible goal for a browser plugin, using cookie-based tracking and communication with a central server to coordinate information sharing across multiple 
user clients. Figure 1 shows one portion of the design, the display of multiple forms of audience awareness information in the context of an existing photo page on Flickr.

While space prevents a full account of all our design decisions, several key features can be highlighted. First, audience information appears directly in multiple use contexts on the system, such as individual photo pages, photo set page, and so forth, rather than only consolidated into a general stats/log report buried several pages deep. This facilitates more immediate access to audience information. It also allows the individual creators to interpret audience information at a level of granularity appropriate to their individual goals and use models. These goals and models were shown in our preliminary interviews to vary between creators, as well as across points in time for specific individuals.

\section{People viewed in the last 7 days show all 37}

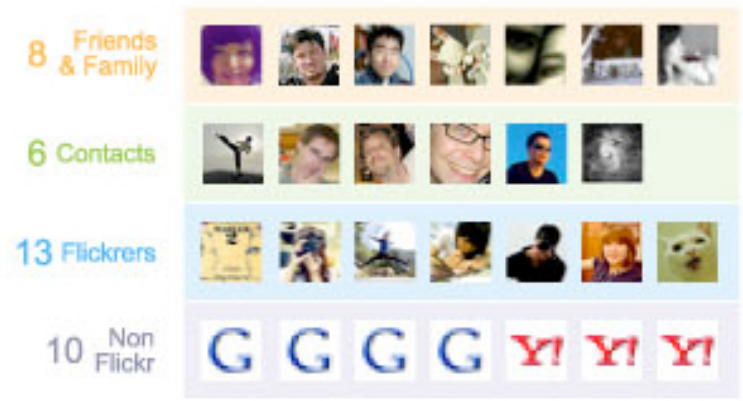

\section{Communities that viewed show all 7}
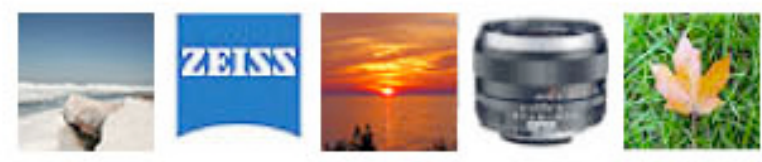

Search Terms show detail / add tags

Huron 2010 Upper Peninsula Great Lakes Lake Fall Break UM
Travel Michigan dawn Autumn Mackinaw Lake Huron

Figure 2: Closeup view of fig.1, showing multiple types audience information, as displayed in the context of a single image's photo page.

Second, the system displays information about multiple audiences concurrently. This is in line with our stated goal of not presupposing a single dominant audience model for any given user a priori. As can be seen in the close up provided in Figure 2, we sought to balance individual audience member detail with information about larger populations. In organizing the display of audiences, we considered both the audience categories from our preliminary interviews and how those audiences would map to the existing privacy control levels, log data and social groupings within Flickr.

We organized the display around degrees of social distance from the creator. The "Friends \& Family" category in the design maps directly to the intimates audience from the interviews, as well as addressing many cases of photo subjects and event participants audience types. The various communities that appeared as audiences in the preliminary interviews are mapped across multiple locations in this interface, contingent on how an individual Flickr user has constructed their contact list. For instance, for some users, professional peers may appear as "contacts."

Communities of interest and practice on Flickr often organize themselves into what are called "groups" in Flickr terminology. We present links from these groups in more community-centric terms to emphasize this notion, labeling these visits as "communities that viewed." We created a visual distinction between individual audience views and links from groups to help the user make inferences about these two different levels of social aggregation. Community may also appear less explicitly, represented by individual users in the "Flickrers" category. Communities external to Flickr, as well as more generalized audiences, are made partially visible both through the "Non Flickr" audience category, as well as through inferences supported by the cloud tag of search terms.

Across all of these audiences, we utilize existing user and group icons to provide visual context. Tooltips for each icon, visible upon mouse-over, provide additional information on user names and group names.

Scaling is one potential limitation of our approach in this design. The number of individuals represented in each audience category can quickly become too large for the available space on an individual photo page, particularly for popular and widely disseminated images. In these cases, the user can click through to a separate page, modeled after Flickr's existing "show all contacts" pages, which present long lists of users. We decided this was a reasonable balance between immediate access to audience information and visual overload on one page. The question of whether this is a sufficient response for all type of photographers remains to be determined in our evaluation exercises.

\section{EVALUATION OF DESIGN}

As mentioned above, our long-term goal for this project is to implement the audience awareness features described above in the form of a Firefox plugin, using cookie-based tracking and communication with a central server to 
coordinate information sharing across multiple user clients. Doing so will allow us to examine emergent practices arising in response to the additional audience awareness and the effects of increased audience awareness on user satisfaction, comfort and creative output.

Prior to proceeding to live deployment, there is a need to verify the assumptions proposed design against a heterogeneous population of photographers. At the time of this submission, this evaluation process is underway, and these results will be featured on our poster. We are utilizing a web-based survey to gather impression data about the proposed design, and begin to see whether it is more effective and appropriate for certain categories of intended audience. The survey focuses on three main categories of data:

a) Demographic information about each photographer.

b) Information about orientations toward photography, including experience level, degree of photographic skill, degree of professional orientation and self-perceived levels of creativity and creative engagement.

c) Perception and attitudes toward different audience conditions, both prior to being exposed to our design mockups and afterwards.

Because our preliminary interviews reinforced the deeply contextualized and personal nature of photo interpretation and many creator/audience interactions, we take a novel approach to customizing the evaluation survey with user's own data. Specifically, we are recruiting existing Flickr users for the survey, and after appropriate consent, we utilize the Flickr API to pull information from their accounts to contextualize a portion of the survey. Rather than showing generic mockups of images with placeholder audiences, we display images from the user's photostream and present them with design mockups populated with plausible audiences, utilizing individuals from their own contact list. We anticipate this contextualized approach to combine advantages from qualitative photo elicitation approaches (Harper, 2002) with those of larger population survey instruments.

\section{ACKNOWLEDGMENTS}

Funding for this project was provided by the National Science Foundation, grant \#IIS0855865.

\section{REFERENCES}

Clark, H. H. (1996). Common ground. In Using language. Cambridge: Cambridge University Press.

Clark, H. H., \& Brennan, S. E. (1991). Grounding in communication. In L. B. Resnick, J. M. Levine, \& S. D. Teasley (Eds.), Perspectives on Socially Shared Cognition (p. 127-149). Washington DC: American Psychological Association.

Cook, E. C., Teasley, S. D. (2011). Beyond Promotion and Protection: Creators, Audiences and Common Ground in User-Generated Media. Proceedings of the 2011 iConference. (Seattle, WA, USA, Feb. 8 - 11, 2011).

Cook, E. C. (2011). Biography, Well-being and Personal Media: A Qualitative Study of Everyday Digital Photography Practices. Dissertation. University of Michigan. Accessed: http://www.simulated.net/ECCookDissertation-Final.pdf.

Erickson, T. \& Kellogg, W. A. (2000) Social Translucence: An Approach to Designing Systems that Support Social Processes. ACM Transactions on Computer-Human Interaction, Vol. 7, No. 1, March 2000, 59-83.

Fussell, S. R. \& Krauss, R. M. (1989) Understanding friends and strangers: The effects of audience design on message comprehension. European Journal of Social Psychology, vol. 19, 509-525.

Harper, D. (2002). Talking about pictures: a case for photo elicitation. Visual Studies, 17, 1. 13-26.

Mitchell, W. J., et al (Eds.). (2003). Beyond Productivity: Information Technology, Innovation and Creativity. Washington DC: National Academies Press.

Newman-Norlund, S. E., et al (2009) Recipient design in tacit communication. Cognition, 111, 46-54.

Nickerson, R. S. (1999). How we know - and sometimes misjudge - what others know: imputing one's own knowledge to others. Psychological Bulletin, 125(6), 737 759.

Shneiderman, B., Fischer, G. et al. (2005). NSF Workshop Report on Creativity Support Tools. June 13 -14, 2005. Accessed: http://www.cs.umd.edu/hcil/CST/report.html. 